\title{
Electrostatically Transduced Face-Shear Mode Silicon MEMS Microresonator
}

\author{
Angel T-H. Lin, Jize Yan, Ashwin A. Seshia \\ Department of Engineering \\ University of Cambridge \\ Cambridge, United Kingdom \\ Email: aas41@cam.ac.uk
}

\begin{abstract}
Silicon microresonators are increasingly viewed as attractive candidates for a variety of frequency selective signal processing applications due to miniaturization and potential for integration with CMOS. In this work, we present a new electrostatically transduced face-shear (FS) mode square plate single crystal silicon resonator that rivals previously reported bulk mode resonator topologies and demonstrates good frequency scaling. A microfabricated face-shear mode resonator with $800 \mu \mathrm{m}$ side length demonstrates a resonant frequency of $3.638 \mathrm{MHz}, Q$ of 11193 in air and 836283 in vacuum as well as a TCF of $-19 \mathrm{ppm} / \mathrm{K}$.
\end{abstract}

\section{INTRODUCTION}

Silicon MEMS resonators are viewed as promising alternative to quartz crystals for a variety of frequency selective signal processing and sensing applications due to its small form factor and potential for integration with CMOS. In particular, bulk mode resonators are widely studied due to their higher quality factor over the flexure modes, and the dimensions scales well with frequency. Previously reported electrostatically transduced square plate bulk modes include the square-extensional (SE) mode [1-3] and the Lamé mode [2-4]. Another type of bulk mode that exists in the square plate is the face shear mode, which has been reported in quartz crystals [5], but has not yet studied in single crystal silicon. In this work, we present an electrostatically transduced faceshear (FS) mode single crystal silicon resonator that rivals previously reported bulk mode resonator topologies. The aim of this work is to study the FS mode and compare the results with those of the SE and Lamé mode. The three mode shapes of interest are illustrated in Fig. 1.
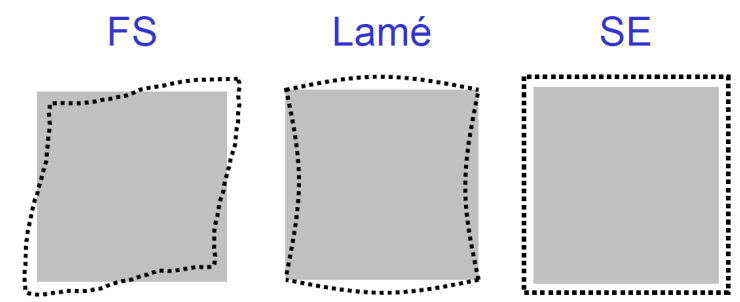

Figure 1. Diagram illustrating three different square plate bulk modes

\section{DEVICE DESCRIPTION AND OPERATION}

The resonator consists of a microfabricated silicon square plate, which is centrally anchored at each of the four sides via a T-shaped anchor. The square plate has a side length of $800 \mu \mathrm{m}$, thickness of $25 \mu \mathrm{m}$, and is electrostatically transduced by electrodes located at the four corners of the square, as shown in Fig. 2. The face-shear mode shape can be described as a square that extends diagonally on one axis in the plane of vibration, while also simultaneously contracts along an orthogonal axis in the same plane. The ANSYS FE simulation of the face-shear (FS) mode shape is shown in Fig. 3a, where maximum displacement occurs at the four corners. T-shaped anchors allow the same resonator to be excited in the SE mode, as shown in the ANSYS simulation in Fig. 3b, where the four sides of the square extends and contracts symmetrically.

The resonant frequency of the face-shear mode may be given by the following relation:

$$
f_{0}=\frac{C_{0}}{L} \sqrt{\frac{G}{\rho}}
$$

where $L, G, \rho, C_{0}$ are the side length of square, shear modulus, density of silicon and the constant frequency parameter respectively. The shear modulus can have a value ranging from $51 \mathrm{GPa}$ to $80 \mathrm{GPa}$ on the (100) plane depending on the direction of shear [6]. In our work we have aligned the sides of the square to the [110] direction where the shear modulus for the FS mode is around $51 \mathrm{GPa}$. The value of $C_{0}$ is extracted to be around 0.62 from ANSYS simulation, and this is within $1 \%$ difference from the experimental value.

The resonator is capacitively transduced using a two-port measurement setup, as shown in Fig. 4. The resonator body is grounded, while DC and AC voltages are applied to the actuation electrodes, which generates a time varying electrostatic force that drives the resonator into motion. A separate DC bias is applied to the sense electrodes for the detection of the motional signal. As small changes in the resonator motion alter the air gap between the resonator and the sense electrode, this induces changes in motional current 
through the air gap whose amplitude peaks at the mechanical resonant frequency. The phase of the electrostatic force and the measured motional current is determined by the polarity of the applied DC bias. Therefore, by inverting the polarity of either the drive or the sense electrode, both FS and SE modes of vibration can be measured using the same electrode configuration, similar to that reported by Lee et al [2].

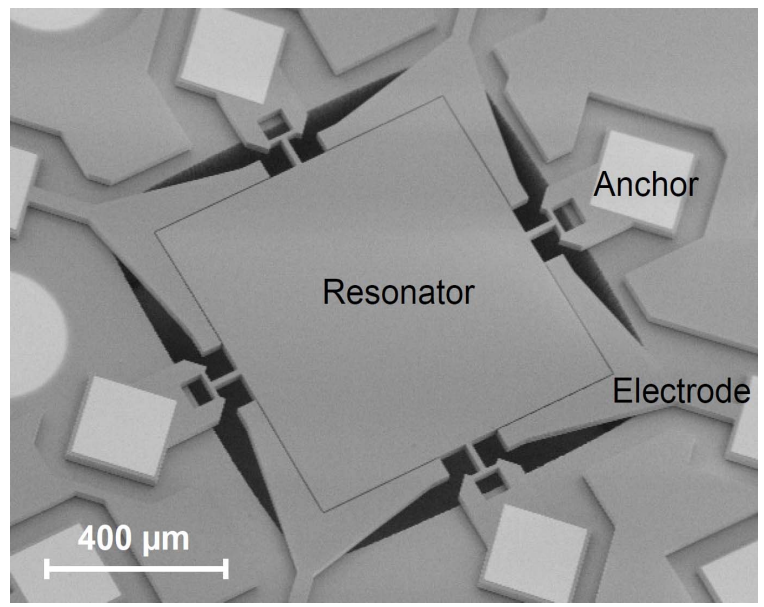

Figure 2. SEM image of the fabricated square plate resonator

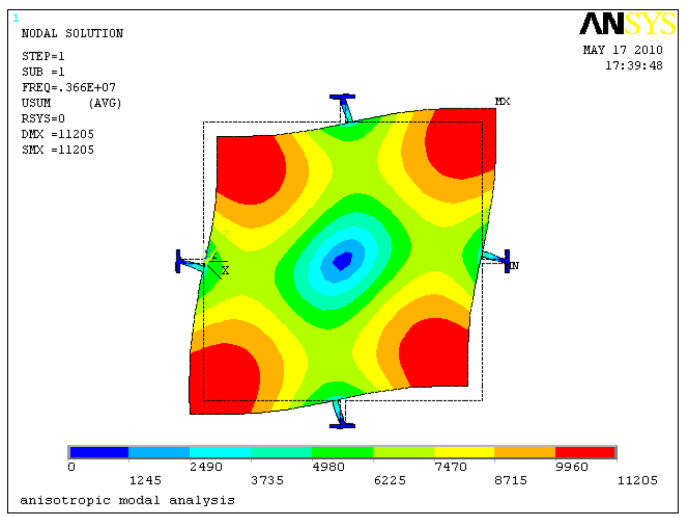

(a)

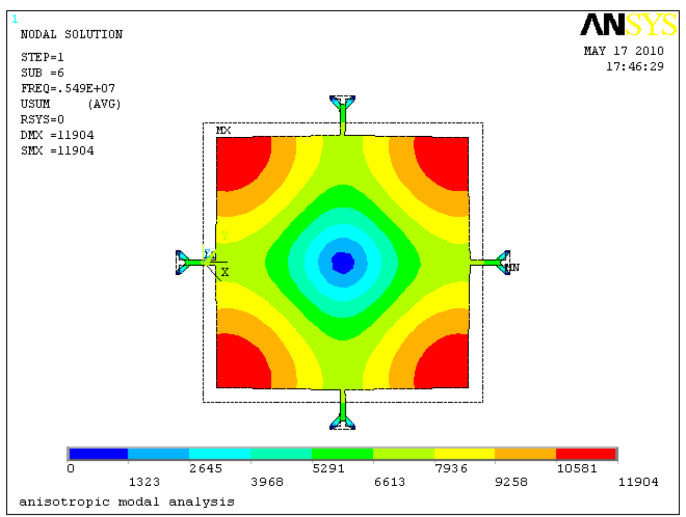

(b)

Figure 3. ANSYS FE simultion of the (a) face shear and (b) squareexntional mode shape.

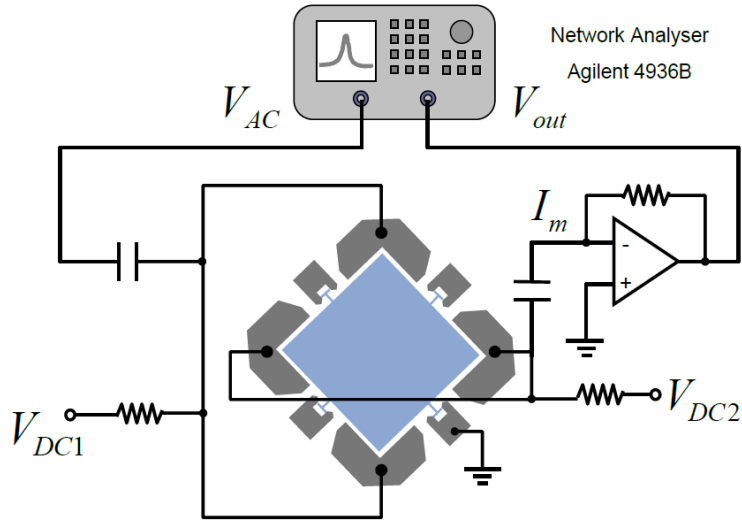

Figure 4. Schematic showing the two-port capacitive setup for the measurement of the face-shear and square-extensional mode.

\section{EXPERIMENTAL RESULTS}

The frequency responses of the FS and SE mode were measured in both vacuum and in air, with the extracted response curves shown in Figs. 5 and 6 . The FS mode resonator demonstrated a resonant frequency of $3.638 \mathrm{MHz}, \mathrm{Q}$ of 11193 in air and 836283 in 10 mTorr vacuum. The same square plate is also independently excited in the squareextensional (SE) mode, by inverting the polarity of the DC actuation voltage on the same electrode configuration. The SE mode demonstrated a resonant frequency of $5.454 \mathrm{MHz}, \mathrm{Q}$ of 9916 in air and 33055 in vacuum. The lower Q for the SE mode is mainly due to anchor losses, as the T-anchor dimensions were not optimized for the SE mode. The ideal situation would be to anchor the square in the centre, which is the nodal point for both the SE and FS mode.

In order to compare the results to a Lamé mode resonator, a separate resonator which is anchored at the 4 corners is used for measuring the Lamé mode response. The Lamé mode shape can be described as a square that contracts along one axis while simultaneously extending along an orthogonal axis in the same plane.

Fig. 7 illustrates the anchor positions for the two resonators. The side anchored resonator enables the excitation of both FS and SE mode, while the corner anchored resonator enables the excitation of both Lamé and SE mode. The same anchor dimensions were used for the two resonators.

The summary of results for all three modes of vibration for the side anchored and cornered anchored square plate are shown in Table 1. The Q factor for the FS and Lamé mode is similar in air and the energy loss is dominated by fluid damping. In vacuum, the $\mathrm{Q}$ factor for Lamé mode is about twice that of the FS mode. This is mainly attributed to the anchor loss effect. In the case of the Lamé mode, the nodal points is located at the 4 corners, therefore the anchoring points have minimal displacement resulting in less energy dissipated via the anchor into the substrates. However, in the case of FS mode, although edge displacements are smaller at the centre edges, it is not a nodal point, as seen from Fig. 3a, and some energy is dissipated via the anchors into the 
substrate, resulting in lower Q as compared to the Lamé mode. The motional resistance of the Lamé mode is also smaller than that of the FS mode, due to the higher quality factor. Similarly the SE mode has the lowest Q and highest motional resistance due to the higher anchor losses, as the T-shaped anchors are not optimally designed for the SE mode. If the anchors can be placed at the centre of the square plate, which is the nodal point for all three bulk modes, the Q-factors of the three modes could be better compared.

An advantage of the FS mode over the Lamé and SE mode is the lower measurement frequency for the same resonator dimensions and transduction area, as it enables stronger transduction of the motional signal in the presence of capacitive feedthrough. This is useful in sensing applications, where higher operating frequencies are not necessarily needed, but a stronger transduction signal in air or liquid is desirable.
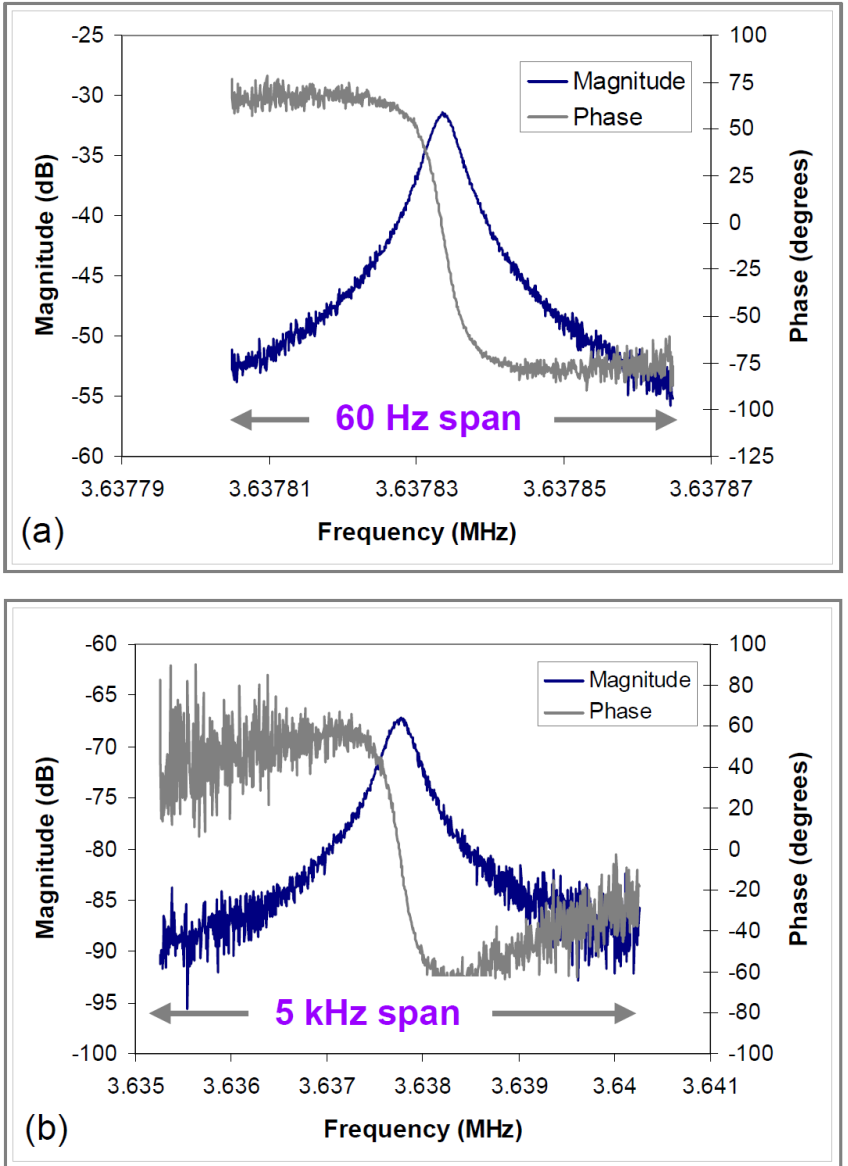

Figure 5. Measured frequency response for the face-shear mode side anchored resonator in (a) vacuum and (b) air.
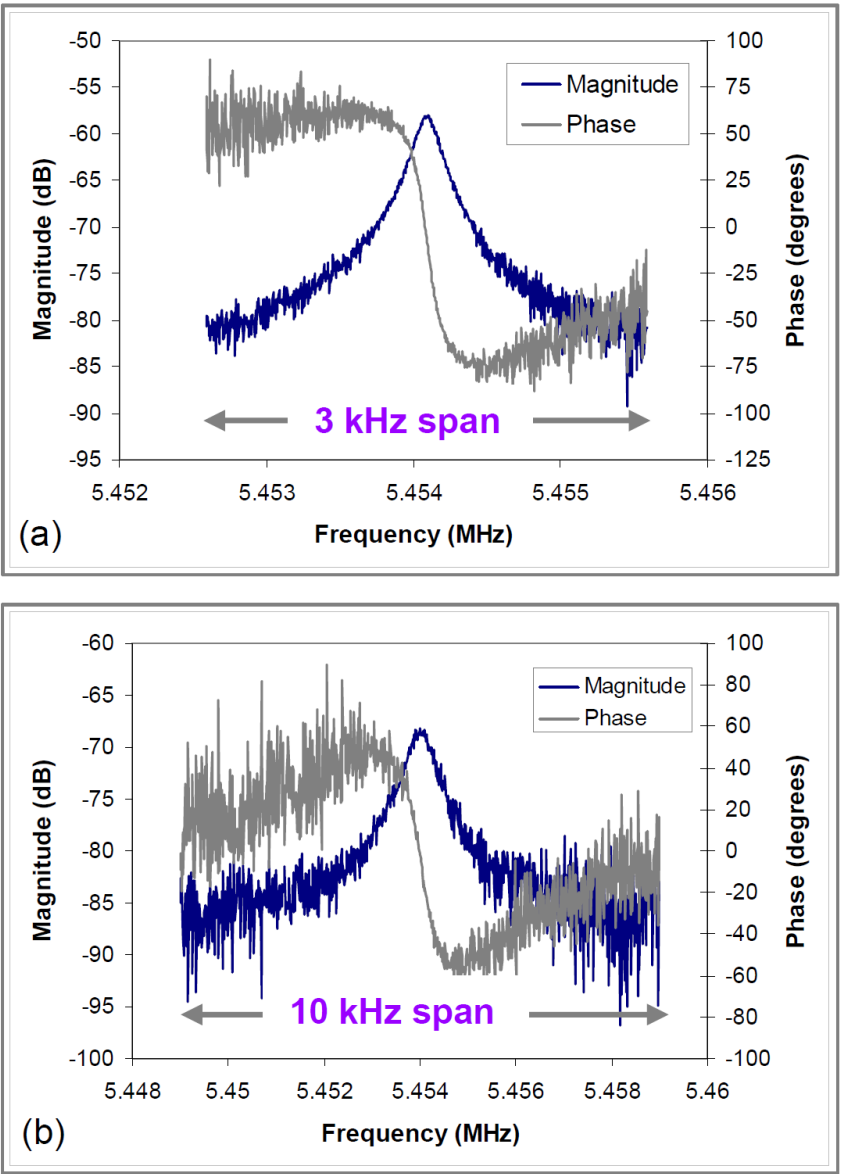

Figure 6. Measured frequency response for the square-extensional mode side anchored resonator in (a) vacuum and (b) air.

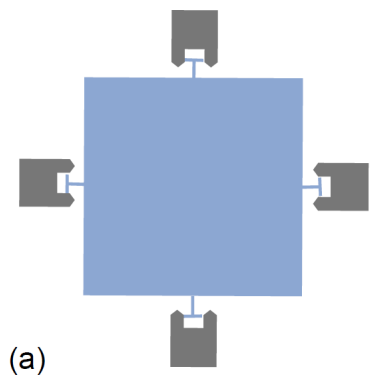

(b)

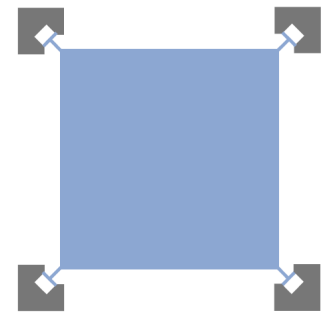

Figure 7. Diagram illustrating the position of the anchors for (a) side anchored square plate and (b) corner anchored square plate. 
TABLE I.

RESULTS SUMMARY

\begin{tabular}{|c|c|c|c|c|}
\hline & \multicolumn{2}{|c|}{ Side anchored } & \multicolumn{2}{c|}{ Corner anchored } \\
\cline { 2 - 5 } & $\boldsymbol{F S}$ & $\boldsymbol{S E}$ & Lamé & $\boldsymbol{S} \boldsymbol{E}$ \\
\hline $\boldsymbol{f}_{\boldsymbol{0}}(\boldsymbol{M H z})$ & 3.638 & 5.454 & 5.162 & 5.463 \\
\hline $\boldsymbol{Q}_{\boldsymbol{A I R}}$ & 11193 & 9916 & 12515 & 10660 \\
\hline $\boldsymbol{Q}_{\boldsymbol{V} \boldsymbol{y} \boldsymbol{C}}$ & 836283 & 33055 & 1652024 & 48564 \\
\hline $\boldsymbol{R}_{\boldsymbol{m}(\mathbf{v a c})}(\boldsymbol{M \Omega})$ & 0.57 & 12.3 & 0.41 & 12.8 \\
\hline
\end{tabular}

\section{TEMPERATURE EFECT}

The variation in resonator frequency with temperature is caused by the thermal expansion of the material and the temperature dependence of the Young's modulus, with the latter being the dominating effect. To investigate the temperature sensitivity of each of the mode shapes, we placed the resonators in a temperature controlled vacuum chamber, and varied the temperature from $300 \mathrm{~K}$ to $360 \mathrm{~K}$. Frequency responses were recorded and lines were fitted through the measured data to extract the temperature coefficient of frequency (TCF) for each mode. Figure 8 shows the measured downshift in frequency with increasing temperature. For the side anchored resonator, the extracted temperature coefficient of frequency for the FS mode is $\alpha_{F S}=((d f / f) / d T)=-19 \mathrm{ppm} / K$ and for the SE mode is $\alpha_{S E}=((d f / f) / d T)=-25.1 \mathrm{ppm} / \mathrm{K}$.

The difference in the temperature coefficient for the FS and SE mode may be due to the fact that the temperature dependence of the elastic stiffness is different in each direction, hence for a FS mode which is dependent on the shear modulus, or the $\mathrm{C}_{66}$ direction, the temperature sensitivity is smaller in the direction of shear as compared to the direction of extension of the SE mode. As for the corner anchored resonator, the extracted temperature coefficient for both Lamé and SE mode were the same, and has a value of $-27 \mathrm{ppm} / \mathrm{K}$.

The electrode configuration as shown in Fig. 4 enables alternating excitation of the two modes of vibration by DC polarity inversion. A resonator that have different TCF for the two vibration modes can potentially be exploited for temperature compensation [3], with the advantage that no external temperature sensor is needed, and the measurement is in-situ therefore there is no error arising from temperature difference between the resonator and the external sensor. The fact that the frequencies of the FS mode and the SE mode are far apart (further than those of the Lamé and SE mode) allows for easier system level implementation as there would be less problems of mode coupling with each other when trying to excite the two modes consecutively.
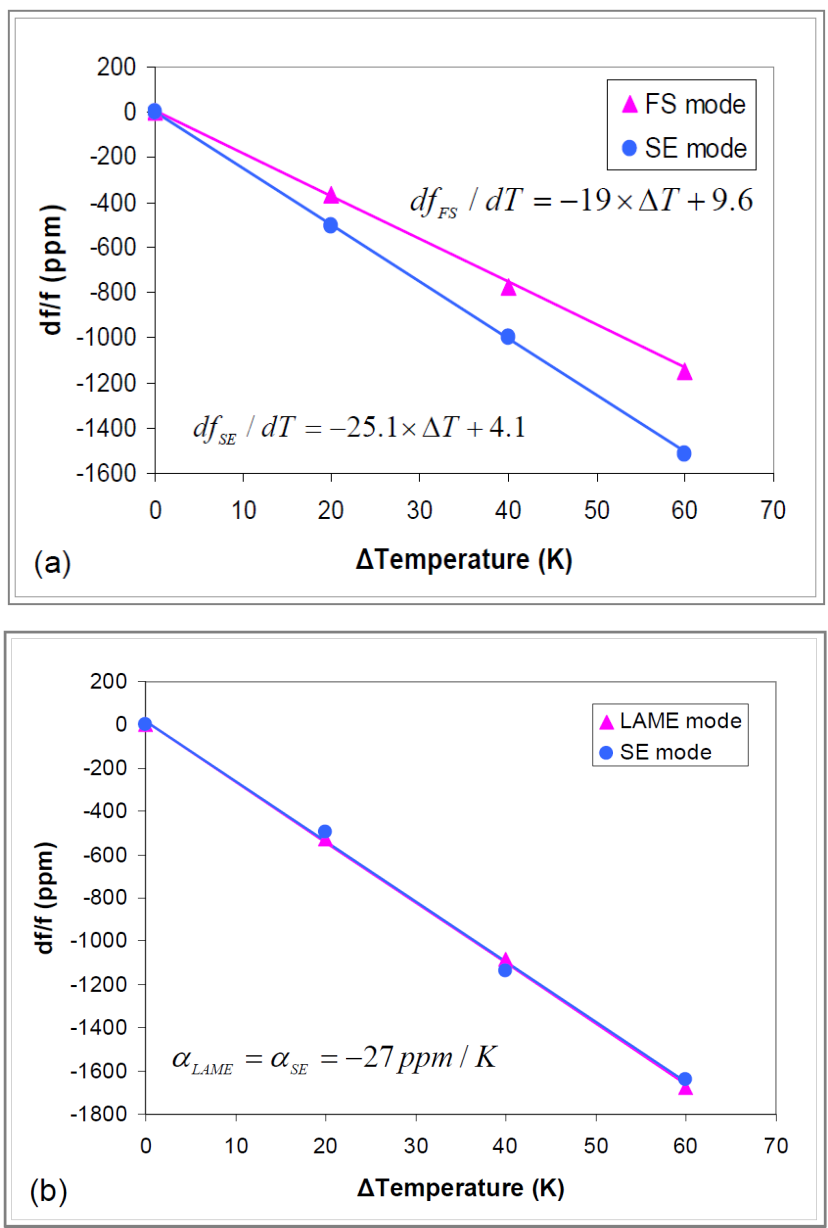

Figure 8. Graph showing the downshift in frequency with increasing temperature for (a) side anchored resonator and (b) corner anchored resonator.

\section{CONCLUSIONS}

In this work, a high quality factor face-shear mode square plate resonator is demonstrated using electrostatic actuation and sensing. The FS mode has a lower TCF relative to the Lamé and SE mode when aligned to the [110] direction on the (100) plane. The lower measurement frequency of the FS mode also enables stronger transduction of the motional signal in the presence of capacitive feedthrough. The ability to consecutively excite the two modes of vibration having different TCFs using the same electrode configuration can potentially be exploited for temperature compensation, particularly given that the resonant frequencies of the FS and SE modes are further apart than those of the Lamé and SE modes. 


\section{ACKNOWLEDGMENT}

This work was supported by the US Army Soldier Systems Centre. A. Lin is a Cambridge Commonwealth Trust scholar and she thanks Dr. J. Lee for the helpful discussions and designing the Lamé mode device tested here.

\section{REFERENCES}

[1] V. Kaajakari, T. Mattila, A. Oja, J. Kiihamaki and H. Seppa, "Squareextensional mode single-crystal silicon micromechanical resonator for low-phase noise oscillator applications," IEEE Electron. Dev. Lett. 25 173-5, 2004.

[2] J. E.-Y. Lee and A. A. Seshia, "Binary excitation of a high Q-factor bulk acoustic microresonator by actuation po-larity inversion," in Proceedings of ASME IMECE 2008, Boston, Massachusetts, USA, 31 Oct - 6 Nov 2008.
[3] M. Koskenvuori, V. Kaajakari, T. Mattila, and I. Tittonen, "Temperature measurement and compensation based on two vibrating modes of a bulk acoustic mode microresonator", MEMS 2008, Tucson, AZ, pp. 78-81.

[4] J. E.-Y. Lee, J. Yan, and A. A. Seshia, "Low loss HF band SOI wine glass bulk mode capacitive square-plate resonator," Journal of Micromechanics and Microengineering, vol. 19, no. 7, 074003, July 2009

[5] H. Kawashima, M. Matsuyama, M. Nakazato. "A variational analysis of a new shape face shear mode quartz crystal resonator formed by an etching method," Proceedings of the 42nd Annual Frequency Control Symposium, Baltimore, MD, USA, June 1-3, 1988.

[6] J. J. Wortman and R. A. Evans, "Youngs' Modulus, Shear Modulus and Poisson's Ratio in Silicon and Germanium," Journal of Applied Physics, vol. 36, pp. 153-156, 1965. 